$\boldsymbol{u}^{b}$

b UNIVERSITÄT

BERN

Grassmannian frequency of Sobolev dimension distortion

Z. M. Balogh, P. Mattila, and J. T. Tyson

Research Report 2013-08

16.08.2013

Mathematics Institute

University of Bern

Sidlerstrasse 5

CH-3012 Bern

Switzerland

www.math.unibe.ch 


\title{
GRASSMANNIAN FREQUENCY OF SOBOLEV DIMENSION DISTORTION
}

\author{
ZOLTÁN M. BALOGH, PERTTI MATTILA, AND JEREMY T. TYSON
}

In memory of Frederick W. Gehring

\begin{abstract}
Let $f$ be a supercritical Sobolev map from a domain $\Omega$ in $\mathbb{R}^{n}$ into a complete separable metric space. For a fixed integer $m, 0<m<n$, and $\alpha>m$, we estimate from above the Hausdorff dimension of the set of elements $V$ in the Grassmannian $G(n, m)$ (equipped with a Riemannian metric) such that $f(V \cap \Omega)$ has positive $\alpha$-dimensional Hausdorff measure. The proof relies heavily on the homogeneous structure of both $G(n, m)$ and the Stiefel manifold $O(n, m)$ of orthogonal injections of $\mathbb{R}^{m}$ into $\mathbb{R}^{n}$. A novel feature of the proof is a Morrey-Sobolev type embedding theorem on the product manifold $O(n, m) \times \mathbb{R}^{m}$, valid for Sobolev maps which factor through the evaluation $\operatorname{map} \Phi: O(n, m) \times \mathbb{R}^{m} \rightarrow \mathbb{R}^{n}, \Phi(\pi, x)=\pi(x)$.
\end{abstract}

2010 Mathematics Subject Classification. Primary: 46E35; Secondary: 28A78, 46E40, 30L99, 31B15, 51M35, 53C30.

Keywords and Phrases. Hausdorff dimension, Sobolev mapping, potential theory, Grassmannian manifold, Stiefel manifold.

\section{INTRODUCTION}

This article contributes to a recent series of papers [3], [4], [5], [15], [7] on the quantitative dimension distortion of Sobolev maps acting on generic members in parameterized families of subsets of their domain.

The historical origins of this research program lie in the theory of quasiconformal mappings, and especially, in the foundational work done by Frederick Gehring in the 1960's. Gehring's signature theorem on the ACL property of quasiconformal maps, [9], is largely responsible for the extensive development which has subsequently occurred, both in the internal theory of quasiconformal maps on Euclidean space and in general metric contexts, as well as in the striking external applications of that theory in areas such as Riemannian geometry, geometric group theory, and dynamics.

The absolute continuity of Euclidean quasiconformal maps, or more generally of Sobolev maps, along lines implies that the image under such a map $f$ of almost every line parallel to a given direction $v \in \mathbb{S}^{n-1}$ is (locally) rectifiable and hence has Hausdorff dimension at most one. Here "almost every" refers to the Lebesgue measure on the orthogonal complement $v^{\perp}$, or equivalently, to the Hausdorff $(n-1)$-measure on $v^{\perp}$.

In [11], Gehring and Väisälä gave sharp, quantitative bounds for the distortion of dimension by quasiconformal maps. The precise formula for these bounds involves the exponent $p(n, K)$ of higher

Date: August 10, 2013.

Z.M.B. supported by the Swiss National Science Foundation, European Research Council Project GALA and European Science Foundation Project HCAA. P.M. supported by the Academy of Finland and the Mathematisches Institut, Universität Bern. J.T.T. supported by US National Science Foundation Grants DMS-0901620 and DMS1201875 . 
integrability for $K$-quasiconformal maps and reads as follows: for a subset $E$ of a domain $\Omega \subset \mathbb{R}^{n}$, $n \geq 2$, and a $K$-quasiconformal map $f: \Omega \rightarrow \mathbb{R}^{n}$, one has

$$
\left(1-\frac{n}{p(n, K)}\right)\left(\frac{1}{\operatorname{dim} E}-\frac{1}{n}\right) \leq \frac{1}{\operatorname{dim} f(E)}-\frac{1}{n} \leq\left(1-\frac{n}{p(n, K)}\right)^{-1}\left(\frac{1}{\operatorname{dim} E}-\frac{1}{n}\right),
$$

or equivalently,

$$
0<\frac{(p(n, K)-n) s}{p(n, K)-s} \leq \operatorname{dim} f(E) \leq \frac{p(n, K) \operatorname{dim} E}{p(n, K)-n+\operatorname{dim} E}<n .
$$

According to Gehring's celebrated higher integrability theorem [10], the quantity

$$
p(n, K):=\sup \left\{p: \text { every } K \text {-quasiconformal } f \text { between domains in } \mathbb{R}^{n} \text { is in } W_{\text {loc }}^{1, p}\right\}
$$

is strictly greater than $n$, for each $n \geq 2$. The exact value of $p(n, K)$ remains a well-known open problem in dimensions $n \geq 3$; when $n=2$ the value $p(2, K)=2 K /(K-1)$ was obtained by Astala [2], with concomitant implications for dimension distortion.

It is nowadays well understood that the requirement of quasiconformality is unnecessary for the upper bound in (1.1), which indeed holds for arbitrary supercritical Sobolev mappings. More precisely, if $E \subset \Omega \subset \mathbb{R}^{n}$ has $\sigma$-finite $s$-dimensional Hausdorff measure for some $s<n$ and $f$ is a $W^{1, p}$ mapping with $p>n$ defined on $\Omega$, then $f(E)$ is a null set for the $(p s) /(p-n+s)$-dimensional Hausdorff measure. See Kaufman [17] for a proof of this fact as well as results demonstrating its sharpness.

The following result from [3] extends the preceding observations to subspaces of arbitrary dimension and to the full spectrum of Hausdorff measures. Here and henceforth in this paper, for integers $0<m<n$ we denote by $G(n, m)$ the Grassmannian manifold consisting of all $m$-dimensional linear subspaces of $\mathbb{R}^{n}$. For a point $a$ in the orthogonal complement $V^{\perp}$ of $V \in G(n, m)$, we denote by $V_{a}=V+a$ the affine subspace parallel to $V$ and passing through $a$. See section 4 for the definition of the metric space-valued Sobolev space $W^{1, p}(\Omega: Y)$.

Theorem 1.1. Let $f \in W^{1, p}(\Omega, Y)$ be a Sobolev map from a domain $\Omega \subset \mathbb{R}^{n}$ to a complete separable metric space $Y$, where $p>n$. Let $V \in G(n, m)$ for some $m$ with $0<m<n$. For each $\alpha$ such that

$$
m<\alpha \leq \frac{p m}{p-n+m}
$$

we have

$$
\mathcal{H}^{\alpha}\left(f\left(V_{a} \cap \Omega\right)\right)=0
$$

for $\mathcal{H}^{\beta}$-almost every $a \in V^{\perp}$, where

$$
\beta=\beta(p, \alpha)=(n-m)-\left(1-\frac{m}{\alpha}\right) p .
$$

The value of $\beta(p, \alpha)$ in Theorem 1.1 is best possible. Note that $\beta(p, \alpha)=0$ if and only if $\alpha=p m /(p-n+m)$; recalling that $\mathcal{H}^{0}$ is the counting measure, one observes that Theorem 1.1 recovers the universal dimension upper bound discussed previously.

The proof of Theorem 1.1 takes advantage of the rectilinear product structure inherent to its formulation, to wit, the decomposition $\mathbb{R}^{n}=V \oplus V^{\perp}$. This simple geometric structure greatly simplifies the analysis. For instance, the fibers $V_{a}$ are disjoint and hence no interference between fibers arises in the control of the dimensions of their images under $f$. Moreover, the rigid product structure is perfectly adapted to the decomposition of $\mathbb{R}^{n}$ into dyadic cubes, and the proof runs efficiently via the sharp Morrey-Sobolev embedding theorem for cubes and the equivalence of Hausdorff and dyadic Hausdorff measures.

In this paper we address the following problem, which was explicitly posed in [3, Problem 6.1]. 
Question 1.2. Given $n, m, p$ and $\alpha$ as in the statement of Theorem 1.1, how large can be a collection of subspaces in $G(n, m)$, all of whose dimensions are increased to $\alpha$ by a $W^{1, p}$ Sobolev mapping?

The Grassmannian $G(n, m)$ is a smooth manifold of dimension $m(n-m)$. It admits a transitive action by the orthogonal group $O(n)$, leading to the standard representation of $G(n, m)$ as a homogeneous space:

$$
G(n, m) \simeq O(n) / O(m) \times O(n-m) .
$$

In addition to its standard Riemannian metric, $G(n, m)$ admits several geometrically natural, mutually comparable metrics, with respect to each of which $O(n)$ acts by isometries. We can make Question 1.2 more precise by asking for estimates for the Hausdorff dimension of that collection of subspaces with respect to any one of these mutually comparable metrics. We review metrics on Grassmannians in section 2.

We now state our main theorem.

Theorem 1.3. Let $f \in W^{1, p}(\Omega, Y)$ be a Sobolev map from a domain $\Omega \subset \mathbb{R}^{n}$ to a complete separable metric space $Y$, where $p>n$. Let $m$ be an integer with $0<m<n$ and let $\alpha$ satisfy (1.2). Then the Grassmannian exceptional set

$$
E_{\alpha}:=\left\{V \in G(n, m): \mathcal{H}_{Y}^{\alpha}(f(V \cap \Omega))>0\right\}
$$

is a null set for the $\gamma$-dimensional Hausdorff measure on $G(n, m)$, where

$$
\gamma=\gamma(p, \alpha)=m(n-m)-\left(1-\frac{m}{\alpha}\right) p .
$$

Observe that the value for $\gamma$ in (1.6) is analogous to that for $\beta$ in (1.4), giving an improved bound relative to the dimension of the parameterizing space $\left(n-m=\operatorname{dim} V^{\perp}\right.$ in Theorem 1.1; $m(n-m)=\operatorname{dim} G(n, m)$ in Theorem 1.3). In the case $m=1$, the Grassmannian $G(n, 1)$ coincides with the real projective space $P_{\mathbb{R}}^{n-1}$, and the values of $\beta$ and $\gamma$ agree. In fact, Theorem 1.3 follows immediately from Theorem 1.1 in the special case $m=1$ by working in charts on $P_{\mathbb{R}}^{n-1}$.

For $m \geq 2$ we cannot derive Theorem 1.3 directly from Theorem 1.1 as the parameterizing Grassmannian $G(n, m)$ has dimension strictly larger than $n-m$. Instead, we lift the problem from $G(n, m)$ to the Stiefel manifold $O(n, m)$ of orthogonal injections of $\mathbb{R}^{m}$ into $\mathbb{R}^{n}$. This is done via the representation $G(n, m) \simeq O(n, m) / O(m)$, where $O(m)$ acts on $O(n, m)$ by precomposition. The various metrics on $G(n, m)$ described above lift to $O(n)$ invariant metrics on $O(n, m)$, and Theorem 1.3 follows directly from

Theorem 1.4. Let $f \in W^{1, p}(\Omega, Y), m$ and $\alpha$ be as in Theorem 1.3. Then the Stiefel exceptional set $F_{\alpha}:=\left\{\pi \in O(n, m): \mathcal{H}_{Y}^{\alpha}\left(f\left(\pi\left(\mathbb{R}^{m}\right) \cap \Omega\right)>0\right\}\right.$ is a null set for the $(\gamma+\operatorname{dim} O(m))$-dimensional Hausdorff measure on $O(n, m)$, where $\gamma$ is defined as in (1.6).

The Stiefel manifold $O(n, m)$ is an $O(m)$ bundle over $G(n, m)$. In particular, $O(n, m)$ has dimension equal to

$$
N:=\operatorname{dim} G(n, m)+\operatorname{dim} O(m)=m(n-m)+\frac{1}{2} m(m-1) .
$$

In order to derive Theorem 1.3 from Theorem 1.4, we use the bundle structure of $O(n, m)$ over $G(n, m)$ to show that $F_{\alpha}=E_{\alpha} \times O(m)$ (locally), and hence $\operatorname{dim} F_{\alpha}=\operatorname{dim} E_{\alpha}+\operatorname{dim} O(m)$.

In Theorem 1.4 the role of the rectilinear decomposition $\mathbb{R}^{n}=V \oplus V^{\perp}$ and the foliation of $\mathbb{R}^{n}$ by the fibers $V_{a}, a \in V^{\perp}$, has been replaced by the product space $O(n, m) \times \mathbb{R}^{m}$ and the fibers $\pi\left(\mathbb{R}^{m}\right), \pi \in O(n, m)$. Note, however, that the overall dimension $\operatorname{dim} O(n, m)+\operatorname{dim} \mathbb{R}^{m}=N+m$ is typically much larger than $n$. Thus Theorem 1.1, which was formulated in the supercritical case $p>n$, cannot be directly applied. 
In the framework of Theorem 1.1, the subcritical case $(p<n)$ was previously addressed by Hencl and Honzík [15]. In [15, Theorem 1.1], a weak form of the conclusion of Theorem 1.1 is obtained in the case $\alpha \leq p<n$. To wit, the Hausdorff measure statement in (1.3) is replaced by the dimension estimate $\operatorname{dim} f\left(V_{a} \cap \Omega\right) \geq \alpha$, and the conclusion about vanishing $\mathcal{H}^{\beta}$ measure is similarly replaced by a dimension estimate. The result of Hencl and Honzík can be applied towards Theorem 1.4 by working in charts on $O(n, m)$, yielding the stated conclusion at the level of Hausdorff dimension. We obtain a slight improvement by deriving results on the level of the Hausdorff measures. It does not appear that such improvement can be obtained using the methods of [15] (see, for example, the discussion in the second paragraph following the statement of Theorem 1.1 in that reference).

Our proof of Theorem 1.4 is rather different from the argument of Hencl and Honzík, relying heavily on the homogeneous structure of the Grassmannian and Stiefel manifolds. In particular, we make a careful analysis of the evaluation mapping $\Phi: O(n, m) \times \mathbb{R}^{m} \rightarrow \mathbb{R}^{n}$, defined by

$$
\Phi(\pi, x)=\pi x .
$$

We obtain a new Morrey-Sobolev-type embedding theorem for Sobolev maps which factor through the evaluation map (valid even in the 'subcritical' case $p<N+m$ ) by applying the coarea formula and estimates for the Jacobian of $\Phi$. We believe that such estimates, and especially the tools used to obtain them, are of independent interest. In order to obtain the desired precise statement about Hausdorff measure, we also need a strong form of the Morrey-Sobolev embedding with the same domain on both sides of the inequality. To this end, we show that the images of cubes in $O(n, m) \times \mathbb{R}^{m}$ under the evaluation map are John domains.

It is natural to inquire about the sharpness of the estimates in Theorem 1.3 and 1.4. The estimate in Theorem 1.1 is optimal for all choices of the data $n, m, p$ and $\alpha$, cf. Theorem 1.4 in [3]. In particular, as previously mentioned, it is well-known that

$$
\operatorname{dim} f(E) \leq \frac{m p}{p-n+m}
$$

whenever $f \in W^{1, p}(\Omega, Y)$ and $E \subset \Omega$ satisfies $\operatorname{dim} E=m$. Note that $\beta(p, \alpha)=0$ if and only if $\alpha=\frac{m p}{n-m+p}$, where $\beta(p, \alpha)$ is defined in (1.4). On the other hand, the value

$$
\gamma\left(\alpha, \frac{m p}{p-n+m}\right)=(n-m)(m-1)=\operatorname{dim} G(n-1, m-1) .
$$

is suggestive. Intuitively we could think that if $f$ blows up even a single lower dimensional subspace $U$ then this would yield a rather large exceptional set in $G(n, m)$, because then all $m$ dimensional spaces $V$ containing $U$ will be also blown up. Although we are unable to completely implement this idea to obtain a sharp example for all choices of the data, we can give an example valid for $W^{1, p}$ maps with $p$ in some range $\left(n, n+\epsilon_{0}\right)$ which is asymptotically sharp as $p \rightarrow n$. See Example 5.1. The following question remains.

Question 1.5. Is the value $\gamma=\gamma(p, \alpha)$ in Theorem 1.3 best possible?

We conclude this introduction with a brief outline of the paper. In section 2 we recall several well studied metrics on the Grassmannian and Stiefel manifolds. The mutual comparability of these metrics is well known, but we provide a short proof for the sake of completeness. In section 3 we develop a series of technical tools used in the proofs of our main results. In particular, we describe the metric and analytic properties of the evaluation mapping $\Phi$. Section 4 contains the proofs of Theorem 1.4 and its corollary, our main Theorem 1.3. In the concluding section 5 we present an example related to Question 1.5 as discussed in the preceding paragraph. 


\section{Background on Grassmannian and Stiefel manifolds}

In this section we describe several geometrically natural metrics on the Grassmannian manifold

$$
G(n, m)=\left\{V: V \text { an } m \text {-dimensional linear subspace of } \mathbb{R}^{n}\right\}
$$

and the (compact) Stiefel manifold ${ }^{1}$

$$
\begin{aligned}
O(n, m) & =\left\{\pi: \mathbb{R}^{m} \rightarrow \mathbb{R}^{n}:\langle\pi x, \pi y\rangle=\langle x, y\rangle \forall x, y \in \mathbb{R}^{m}\right\} \\
& =\left\{\pi \in \mathbb{R}^{n \times m}: \pi^{T} \pi=\operatorname{id}_{m}\right\} .
\end{aligned}
$$

Here we write $\langle\cdot, \cdot\rangle$ for the standard Euclidean inner product and we identify linear maps $\mathbb{R}^{k} \rightarrow \mathbb{R}^{\ell}$ with $\ell \times k$ matrices.

2.1. Homogeneous structure of Grassmannian and Stiefel manifolds. The group $O(n)$ acts on $O(n, m)$ by postcomposition, and the stabilizer of a point $\pi \in O(n, m)$ identifies with $O(n-m)$ (acting on the orthogonal complement $\operatorname{im}(\pi)^{\perp}$ of the image of $\pi, \operatorname{im}(\pi)$ ). Thus

$$
O(n, m) \simeq O(n) / O(n-m) .
$$

The group $O(m)$ acts on $O(n, m)$ by precomposition, and the orbit of $\pi \in O(n, m)$ under this action identifies with $\operatorname{im}(\pi) \in G(n, m)$. Thus

$$
G(n, m) \simeq O(n, m) / O(m) \simeq O(n) /(O(m) \times O(n-m)) .
$$

Note that when $\pi \in O(n, m)$ with $V=\operatorname{im}(\pi)$, the map $\pi \pi^{T}$ coincides with the orthogonal projection $P_{V}: \mathbb{R}^{n} \rightarrow V$.

In what follows, we denote by $\|A\|_{F}=\sqrt{\operatorname{tr}\left(A^{T} A\right)}$ the Frobenius norm on matrices and by $\|A\|$ the operator norm. We recall the estimates $\|A\| \leq\|A\|_{F} \leq \sqrt{m}\|A\|$ for an $n \times m$ matrix $A$. Note that $\|\pi\|=1$ for all $\pi \in O(n, m)$.

2.2. Metrics on Stiefel manifolds. We first introduce the Frobenius metric

$$
d_{F}(\pi, \rho):=\|\pi-\rho\|_{F}, \quad \pi, \rho \in O(n, m) .
$$

Under the standard identification of $n \times m$ matrices with $\mathbb{R}^{n \times m}, d_{F}$ coincides with the standard Euclidean metric.

The metric $d_{F}$ is well suited to study metric properties of the evaluation map (1.7). However, the internal structure of $O(n, m)$ in this metric is not so clear. For instance, it is not clear whether or not $\left(O(n, m), d_{F}\right)$ is a quasiconvex metric space, or whether balls are connected. For this reason we will also work with a Riemannian distance function $d_{g}$ on $O(n, m)$. Recalling that the tangent space to $O(n, m)$ at a point $\pi_{0}$ is

$$
T_{\pi_{0}} O(n, m)=\left\{B \in \mathbb{R}^{n \times m}: \pi_{0}^{T} B+B^{T} \pi_{0}=0\right\},
$$

we fix the Riemannian metric $g$ on $O(n, m)$ given by

$$
g_{\pi_{0}}\left(B_{1}, B_{2}\right):=\operatorname{tr}\left(B_{1}^{T} B_{2}\right), \quad \pi_{0} \in O(n, m), B_{1}, B_{2} \in T_{\pi_{0}} O(n, m) .
$$

Note that under the identification described above, this corresponds to the induced Riemannian tensor from the ambient Euclidean space $\mathbb{R}^{n \times m}$.

In order to ensure that our conclusions in Theorem 1.4 are well stated, we need to know that this metric is comparable to $d_{F}$. This is the content of the following proposition.

Proposition 2.1. There exists a constant $C>0$ so that $C^{-1} d_{F} \leq d_{g} \leq C d_{F}$.

\footnotetext{
${ }^{1}$ The Stiefel manifold is usually defined as the space of orthonomal $m$-frames in $\mathbb{R}^{n}$. This space is canonically identified with $O(n, m)$ via the bijection $\pi \leftrightarrow\left(\pi\left(\mathbf{e}_{1}\right), \ldots, \pi\left(\mathbf{e}_{m}\right)\right)$, where $\mathbf{e}_{1}, \ldots, \mathbf{e}_{m}$ denotes the standard basis in $\mathbb{R}^{m}$.
} 
Proof. Since $O(n, m)$ is compact, it suffices to prove that these two metrics are equivalent on small scales, e.g., to prove that there exists $\delta>0$ so that the ratio $d_{g}(\pi, \rho) / d_{F}(\pi, \rho)$ is bounded away from zero and infinity for $0<d(\pi, \rho) \leq \delta$. This is proved in Proposition II.1 of [16] under the restriction $m \leq n / 2$. An easy modification of the proof addresses the remaining case $m \geq n / 2 .^{2}$

2.3. Metrics on Grassmannian manifolds. We describe three mutually comparable metrics on the Grassmannian $G(n, m)$. Each of these metrics admits an explicit formula in terms of the so-called principal angles between two $m$-dimensional subspaces. We first recall this concept.

Fix elements $V, W \in G(n, m)$. Choose unit vectors $v_{1} \in V$ and $w_{1} \in W$ such that $\left\langle v_{1}, w_{1}\right\rangle=$ $\min \langle v, w\rangle$ where the minimum is over all unit vectors $v \in V, w \in W$. Next, choose unit vectors $v_{2} \in V$ and $w_{2} \in W$ such that $\left\langle v_{2}, w_{2}\right\rangle=\min \langle v, w\rangle$, where this time the minimum is over all unit vectors $v \in V$ with $\left\langle v, v_{1}\right\rangle=0$ and $w \in W$ with $\left\langle w, w_{1}\right\rangle=0$. Continuing inductively, define $v_{j}$ and $w_{j}$ for all $j=1, \ldots, m$. The vectors $v_{1}, \ldots, v_{m}$ and $w_{1}, \ldots, w_{m}$ are the principal vectors associated to the pair $V, W$, and the angles $\theta_{j}=\arccos \left\langle v_{j}, w_{j}\right\rangle$ are the corresponding principal angles.

The principal angles between elements in $G(n, m)$ can be efficiently computed in terms of representing transformations in $O(n, m)$. Let $\pi, \rho \in O(n, m)$ and consider a singular value decomposition

$$
\pi^{T} \rho=U D \tilde{U}^{T}
$$

i.e. $U, \tilde{U} \in O(m)$ and $D$ is a diagonal matrix with nonnegative diagonal entries. It is clear that the diagonal matrix $D$ is unaffected by precomposition of either $\pi$ or $\rho$ by orthogonal matrices, hence the entries in $D$ depend only on the image subspaces. In fact, if we assume, as we may without loss of generality, that the diagonal entries $d_{11}, \ldots, d_{m m}$ of $D$ are nonincreasing, then $d_{j j}=\cos \theta_{j}$, $j=1, \ldots, m$, where $\theta_{1}, \ldots, \theta_{m}$ denote the principal angles between $V=\operatorname{im}(\pi)$ and $W=\operatorname{im}(\rho)$.

We refer the reader to [8] for further details.

Procrustes metric. This metric is frequently employed in applications of Grassmannians to statistical shape analysis, see [6] or [12]. It is obtained by a standard quotient construction applied to the Frobenius metric $d_{F}$ on $O(n, m)$. Define the Procrustes metric

$$
\overline{d_{F}}(\pi, \rho):=\inf _{g \in O(m)}\|\pi g-\rho\|_{F},
$$

It is easy to check that $\overline{d_{F}}$ induces a metric on $O(n, m) / O(m) \simeq G(n, m)$; we use the same notation for this metric.

An explicit formula for the Procrustes metric in terms of principal angles is

$$
\overline{d_{F}}(V, W)=2 \sqrt{\sum_{j=1}^{m} \sin ^{2}\left(\theta_{j} / 2\right)} .
$$

Indeed, according to the solution of the classical orthogonal Procrustes problem [12, Chapter 4], the infimum in (2.4) is achieved for $g=U \tilde{U}^{T}$, where $U$ and $\tilde{U}$ are defined via (2.3). Then

$$
\|\pi-\rho g\|_{F}^{2}=2(m-\operatorname{tr} D)=4 \sum_{j} \sin ^{2}\left(\theta_{j} / 2\right)
$$

\footnotetext{
${ }^{2}$ In Appendix B of [16], in the argument following equation (B3), reverse the roles of $k=m$ and $n-k$ and project into a different $k \times k$ submatrix. Note also that the argument in [16] is presented for the complex Stiefel manifold and must be slightly reworded to apply to the real case.
} 
Projector metric. Another natural $O(n)$ invariant metric on $G(n, m)$ arises from the identification of elements $V \in G(n, m)$ with orthogonal projectors $P_{V}: \mathbb{R}^{n} \rightarrow V$. Define

$$
\overline{d_{P}}(V, W):=\left\|P_{V}-P_{W}\right\|_{F} .
$$

We will again use the same notation to denote the corresponding metric on $O(n, m)$, given by

$$
\overline{d_{P}}(\pi, \rho)=\left\|\pi \pi^{T}-\rho \rho^{T}\right\|_{F} .
$$

The projector metric $\overline{d_{P}}$ also admits an explicit expression in term of principal angles:

$$
\overline{d_{P}}(V, W)=\sqrt{2 \sum_{j=1}^{m} \sin ^{2} \theta_{j}} .
$$

The formula in (2.8) can be obtained from (2.7) using the definition of the Frobenius norm and the singular value decomposition (2.3).

Geodesic metric. The Riemanian metric tensor $g$ on $O(n, m)$ defined above is $O(m)$ invariant, and descends to a metric tensor (still denoted $g$ ) on $G(n, m)$. Wong [19] proved that the corresponding geodesic metric is given in terms of principal angles by the explicit formula

$$
\overline{d_{g}}(V, W)=\sqrt{\sum_{j=1}^{m} \theta_{j}^{2}} .
$$

In view of (2.5), (2.8) and (2.9), together with the fact that all principal angles $\theta_{j}$ lie in the interval $[0, \pi / 2]$ (so $\sin \theta_{j} \simeq \theta_{j}$ ), the following proposition is clear.

Proposition 2.2. The Procrustes, projector and geodesic metrics on $G(n, m)$ are mutually comparable. That is there exists $C>0$ so that $C^{-1} d_{1} \leq d_{2} \leq C d_{1}$, where $d_{1}, d_{2} \in\left\{\overline{d_{F}}, \overline{d_{P}}, \overline{d_{g}}\right\}$.

We emphasize that the metrics $d_{F}$ and $\overline{d_{F}}$ on $O(n, m)$ are not comparable. Thus some care must be taken in appropriately formulating statements regarding Hausdorff measure and dimension in the parameter space $O(n, m)$. Note, however, that the quotient map from $\left(O(n, m), d_{F}\right)$ to $\left(G(n, m), \overline{d_{F}}\right)$ is 1-Lipschitz.

\section{Metric And Analytic PRoperties of the EVAluation mapping}

In this section we study the evaluation map $\Phi: O(n, m) \times \mathbb{R}^{m} \rightarrow \mathbb{R}^{n}$ defined in (1.7). The primary metric fact which we will prove is that restrictions of $\Phi$ to spherical shells in the $\mathbb{R}^{m}$ factor are Lipschitz quotient maps. This fact has an analytic counterpart: the Jacobian of $\Phi$ is uniformly bounded away from zero and infinity on such spherical shells. Finally, we show that $\Phi$ is a submersion on $O(n, m) \times\left(\mathbb{R}^{m} \backslash\{0\}\right)$ and use this fact to verify the Ahlfors regularity of its level sets.

Notation. We denote by $B(x, r)$ the closed ball with center $x$ and radius $r$ in a metric space $X$. Unless otherwise specified, we work with the metric $d_{F}$ on the Stiefel manifold $O(n, m)$. We equip $O(n, m) \times \mathbb{R}^{m}$ with the product metric $d_{F} \times d_{\infty}$ (where $d_{\infty}$ denotes the $\ell^{\infty}$ metric on $\mathbb{R}^{m}$ ), we write $p=(\pi, x)$ for points in $O(n, m) \times \mathbb{R}^{m}$, and we consider cubes $Q\left(p_{0}, r\right)=B_{d_{F}}\left(\pi_{0}, r\right) \times B_{d_{\infty}}\left(x_{0}, r\right)$ in $O(n, m) \times \mathbb{R}^{m}$. We write $a \wedge b$, resp. $a \vee b$, for the minimum, resp. maximum, of real numbers $a, b$.

Proposition 3.1. For any $\pi_{0} \in O(n, m)$, for any $0 \neq x_{0} \in \mathbb{R}^{m}$ and for any $0<r \leq 1$, we have

$$
B\left(\pi_{0} x_{0}, \frac{1}{4 \sqrt{m}}\left(1 \wedge\left|x_{0}\right|\right) r\right) \subset \Phi\left(Q\left(p_{0}, r\right)\right) \subset B\left(\pi_{0} x_{0}, 2 \sqrt{n}\left(1 \vee\left|x_{0}\right|\right) r\right)
$$


In the previous proposition we used the Euclidean metric on the target $\mathbb{R}^{n}$.

A map $\Phi: X \rightarrow Y$ between metric spaces is said to be a (local) Lipschitz quotient map with constant $C$ if there exists $r_{0}>0$ so that for any $x \in X$ and any $0<r \leq r_{0}$, we have $B_{Y}\left(\Phi(x), C^{-1} r\right) \subset \Phi\left(B_{X}(x, r)\right) \subset B_{Y}(\Phi(x), C r)$. Proposition 3.1 implies that $\Phi$ is a Lipschitz quotient map on sets $O(n, m) \times\left\{x: L^{-1} \leq|x| \leq L\right\}$.

Proof. The right hand inclusion is proven as follows. If $(\pi, x) \in Q\left(p_{0}, r\right)$, then (recall that $\|A\|$ denotes the operator norm of a matrix $A$, while $|x|$ denotes the Euclidean norm of a point $x$ )

$$
\begin{aligned}
\left|\pi x-\pi_{0} x_{0}\right| & \leq|| \pi||\left|x-x_{0}\right|+|| \pi-\pi_{0}||\left|x_{0}\right| \\
& \leq \sqrt{n}\left\|\pi|| d_{\infty}\left(x, x_{0}\right)+|| \pi-\pi_{0}\right\|_{F}\left|x_{0}\right| \\
& \leq\left(\sqrt{n}+\left|x_{0}\right|\right) r \\
& \leq 2 \sqrt{n}\left(1 \vee\left|x_{0}\right|\right) r
\end{aligned}
$$

as desired. To prove the left hand inclusion, let $y \in \mathbb{R}^{n}$ satisfy $\left|\pi_{0} x_{0}-y\right| \leq(4 \sqrt{m})^{-1}\left(1 \wedge\left|x_{0}\right|\right) r$. Then $|y| \geq\left(1-(4 \sqrt{m})^{-1} r\right)\left|x_{0}\right| \geq \frac{1}{2}\left|x_{0}\right|$. Set $z=t \pi_{0} x_{0}$, where $t$ is chosen so that $|z|=|y|$. Then $z \in B\left(\pi_{0} x_{0},(4 \sqrt{m})^{-1}\left(1 \wedge\left|x_{0}\right|\right) r\right)$. Pick $A \in O(n)$ so that $A z=y$ and $\left\|A-\mathrm{id}_{n}\right\|=\frac{|y-z|}{|z|} \leq r / \sqrt{m}$. Let $\pi=A \pi_{0}$, then $\left\|\pi-\pi_{0}\right\| \leq\left\|A-\operatorname{id}_{n}\right\| \leq r / \sqrt{m}$ and so $\left\|\pi-\pi_{0}\right\|_{F} \leq r$. Finally, let $x=\pi_{0}^{-1} z$, then $d_{\infty}\left(x, x_{0}\right) \leq\left|x-x_{0}\right|=\left|z-\pi_{0} x_{0}\right| \leq r / 4<r$. Hence $y=A z=\pi x$ with $\pi \in B_{d_{F}}\left(\pi_{0}, r\right)$ and $x \in B_{d_{\infty}}\left(x_{0}, r\right)$, i.e., $(\pi, x) \in Q\left(p_{0}, r\right)$.

A domain $\Omega \subset \mathbb{R}^{n}$ is a John domain if there exists $y_{0} \in \Omega$ (the John center) and a constant $A>0$ (the John constant) so that every point $y \in \Omega$ can be joined to $y_{0}$ by a rectifiable curve $\gamma$ with the property that, for each $z \in \gamma$, the length of the subcurve of $\gamma$ from $y$ to $z$ is bounded above by $A \operatorname{dist}(z, \partial \Omega)$. The importance of John domains for us is the fact (see Proposition 4.1) that the Morrey-Sobolev embedding theorem holds on such domains, in the same form as for Euclidean balls.

The following lemma is elementary. For a metric space $X$, let $\mathcal{B}(X)$ denote the collection of all metric balls in $X$.

Lemma 3.2. Let $X$ be a metric space such that all elements of $\mathcal{B}(X)$ are John domains with a universal John constant $A$. If $f: X \rightarrow Y$ is a Lipschitz quotient surjection with constant $C$, then all elements of $\{f(B): B \in \mathcal{B}(X)\}$ are John domains with John constant $C^{2} A$.

Proposition 3.3. For each $L>0, \pi_{0} \in O(n, m), x_{0} \in \mathbb{R}^{m}, L^{-1} \leq\left|x_{0}\right| \leq L$ and $0<r \leq \frac{1}{2}\left|x_{0}\right|$, the set $\Phi\left(Q\left(p_{0}, r\right)\right)$ is a John domain with center $\pi_{0} x_{0}$ and John constant $A$ depending only on $L$, $m$ and $n$.

Proof. Consider $O(n, m) \times \mathbb{R}^{m}$ equipped with the product metric $d_{g} \times d_{\infty}$, where $d_{g}$ denotes the Riemannian metric on $O(n, m)$. This is a geodesic space, hence its metric balls are John domains, with a universal John constant [13, Corollary 9.5]. By Proposition 2.1 and the bi-Lipschitz invariance of the John domain condition, balls in $\left(O(n, m) \times \mathbb{R}^{m}, d_{F} \times d_{\infty}\right)$ are John domains with a universal John constant. We complete the proof by applying the preceding lemma with $X=O(n, m) \times\left\{x: L^{-1} \leq|x| \leq L\right\}, f=\Phi$, and $Y=\left\{y \in \mathbb{R}^{n}: L^{-1} \leq|y| \leq L\right\}$.

In what follows, we write $\left(\mathbb{R}^{n}\right)^{*}:=\mathbb{R}^{n} \backslash\{0\}$ and similarly for $\left(\mathbb{R}^{m}\right)^{*}$.

Proposition 3.4. The evaluation map $\Phi$ is a submersion of $O(n, m) \times\left(\mathbb{R}^{m}\right)^{*}$ to $\left(\mathbb{R}^{n}\right)^{*}$.

Proof. The differential of $\Phi$, at $\pi_{0} \in O(n, m)$ and $0 \neq x_{0} \in \mathbb{R}^{m}$, is $D \Phi_{\left(\pi_{0}, x_{0}\right)}(B, v)=B x_{0}+\pi_{0} v$. Recall that the tangent space to $O(n, m)$ was identified in (2.2). We identify the tangent spaces of $\left(\mathbb{R}^{m}\right)^{*}$ and $\left(\mathbb{R}^{n}\right)^{*}$, with $\mathbb{R}^{m}$ and $\mathbb{R}^{n}$ respectively. In order to show that $D \Phi_{\left(\pi_{0}, x_{0}\right)}$ has full rank, we verify the following stronger statement: 
Claim 3.5. For each $w \in \mathbb{R}^{n}$ and each $B \in T_{\pi_{0}} O(n, m)$, there exists $\widetilde{B} \in T_{\pi_{0}} O(n, m)$ and $v \in \mathbb{R}^{m}$ so that $\pi_{0}^{T} B=\pi_{0}^{T} \widetilde{B}$ and $\widetilde{B} x_{0}+\pi_{0} v=w$.

We consider $\pi_{0}$ as an $n \times m$ matrix, whose columns are orthonormal. Let $\pi_{0}^{\perp} \in O(n, n-m)$ correspond to an $n \times(n-m)$ matrix so that the columns of $\pi_{0}$ and $\pi_{0}^{\perp}$ (taken together) form an orthonormal basis of $\mathbb{R}^{n}$. Then $\pi_{0}^{T} \pi_{0}^{\perp}=0$ while $\left(\pi_{0}^{\perp}\right)^{T} \pi_{0}^{\perp}=\mathrm{id}_{n-m}$.

Let $C=\pi_{0}^{T} B$. By (2.2), $C$ is skew-symmetric. Define

$$
\widetilde{B}:=\pi_{0} C+\frac{1}{\left|x_{0}\right|^{2}} \pi_{0}^{\perp}\left(\pi_{0}^{\perp}\right)^{T} w x_{0}^{T} .
$$

Then

$$
\pi_{0}^{T} \widetilde{B}=C+\frac{1}{\left|x_{0}\right|^{2}} \pi_{0}^{T} \pi_{0}^{\perp}\left(\pi_{0}^{\perp}\right)^{T} w x_{0}^{T}=C=\pi_{0}^{T} B
$$

and (again by (2.2)), $\widetilde{B} \in T_{\pi_{0}} O(n, m)$. Define $v:=\pi_{0}^{T} w-C x_{0}$. Then

$$
\begin{aligned}
\widetilde{B} x_{0}+\pi_{0} v & =\pi_{0} C x_{0}+\frac{1}{\left|x_{0}\right|^{2}} \pi_{0}^{\perp}\left(\pi_{0}^{\perp}\right)^{T} w x_{0}^{T} x_{0}+\pi_{0} \pi_{0}^{T} w-\pi_{0} C x_{0} \\
& =\pi_{0}^{\perp}\left(\pi_{0}^{\perp}\right)^{T} w+\pi_{0} \pi_{0}^{T} w \\
& =P_{\operatorname{im}\left(\pi_{0}^{\perp}\right)}(w)+P_{\operatorname{im}\left(\pi_{0}\right)}(w)=P_{\operatorname{im}\left(\pi_{0}\right)^{\perp}}(w)+P_{\operatorname{im}\left(\pi_{0}\right)}(w)=w .
\end{aligned}
$$

The proof is complete.

It follows from Proposition 3.4 that every point $y$ of $\left(\mathbb{R}^{n}\right)^{*}$ is a regular value of $\Phi$ and so, for all such $y, \Phi^{-1}(y)$ is a smooth submanifold of dimension $N+m-n$. Moreover, $\Phi^{-1}(y)$ is compact, since it is a closed submanifold of $O(n, m) \times \mathbb{S}^{m-1}(|y|)$.

The conclusions in the following proposition do not depend on the choice of metric on $O(n, m) \times$ $\mathbb{R}^{m}$ up to bi-Lipschitz equivalence. For convenience, in the proof we work with the metric

$$
d\left(\left(\pi_{1}, x_{1}\right),\left(\pi_{2}, x_{2}\right)\right)=\| \pi_{1}-\pi_{2}||+\left|x_{1}-x_{2}\right| .
$$

Proposition 3.6. (1) $O(n, m) \times \mathbb{R}^{m}$ is Ahlfors $(N+m)$-regular.

(2) For each $0 \neq y \in \mathbb{R}^{n}, \Phi^{-1}(y)$ is Ahlfors $(N+m-n)$-regular. The Ahlfors regularity constant depends on $|y|$, but remains bounded on spherical shells $\left\{y \in \mathbb{R}^{n}: L^{-1} \leq|y| \leq L\right\}$.

Proof. Every smooth compact Riemannian manifold is Ahlfors regular in its dimension, and products of Ahlfors regular metric measure spaces are Ahlfors regular. These remarks suffice to deduce (1) and the first assertion in (2).

It remains to verify that the Ahlfors regularity constant of $\Phi^{-1}(y)$ remains bounded for $|y|$ bounded away from zero and infinity. We use an invariance property of the evaluation mapping under rotations and scalings. Given $y \neq 0$, there exists $A_{y} \in O(n)$ so that $A_{y}\left(\frac{y}{|y|}\right)=e_{1}$, where $e_{1}$ denotes the first vector in the standard basis of $\mathbb{R}^{n}$. Then the map

$$
(\pi, x) \mapsto\left(A_{y} \pi,|y|^{-1} x\right)
$$

is a bijection from $\Phi^{-1}(y)$ to $\Phi^{-1}\left(e_{1}\right)$. We claim that it is $L$-bi-Lipschitz. Indeed, for $\left(\pi_{1}, x_{1}\right)$ and $\left(\pi_{2}, x_{2}\right)$ in $\Phi^{-1}(y)$

$$
\left\|A_{y} \pi_{1}-A_{y} \pi_{2}\right\|+\left|\frac{x_{1}}{|y|}-\frac{x_{2}}{|y|}\right|=|| A_{y}\left(\pi_{1}-\pi_{2}\right)||+\frac{\left|x_{1}-x_{2}\right|}{|y|} \leq|| \pi_{1}-\pi_{2}||+L\left|x_{1}-x_{2}\right|
$$

and similarly ||$A_{y} \pi_{1}-A_{y} \pi_{2}||+\left|\frac{x_{1}}{|y|}-\frac{x_{2}}{|y|}\right| \geq|| \pi_{1}-\pi_{2}||+L^{-1}\left|x_{1}-x_{2}\right|$. It follows that the Ahlfors regularity constant for $\Phi^{-1}(y)$ can be bounded in terms of $L$ when $L^{-1} \leq|y| \leq L$, as asserted. 
For a linear map $L: V \rightarrow W$ between Hilbert spaces $V$ and $W$ with $\operatorname{dim} V \geq \operatorname{dim} W=n$, we denote by $J_{n} L$ the Jacobian (or $n$-dimensional coarea factor) of $L$, defined as follows:

$$
J_{n}(L)=\sqrt{\operatorname{det}\left(L \circ L^{T}\right)},
$$

where $L^{T}$ denotes the transpose of $L$. For the evaluation map $\Phi$, we abbreviate

$$
(J \Phi)_{p}:=J_{n}\left(D \Phi_{p}\right) .
$$

We will employ the following coarea formula (see, for example, Theorem 2.93 in [1] for the case of Lipschitz mappings and $M=\mathbb{R}^{N}$ ):

Proposition 3.7 (Coarea formula). Let $M$ be a smooth d-dimensional Riemannian manifold, $d \geq n$, let $F: M \rightarrow \mathbb{R}^{n}$ be a smooth map, and let $g: M \rightarrow[0, \infty]$ be Borel. Then

$$
\int_{M} g(x) J_{n}\left(D F_{x}\right) d x=\int_{\mathbb{R}^{n}}\left(\int_{\{F=y\}} g(z) d z\right) d y .
$$

In (3.1) we use the Riemannian volume element on $M$ and on the level sets of $F$. We will apply this formula to the map $\Phi: M \rightarrow \mathbb{R}^{n}$ where $M=O(n, m) \times \mathbb{R}^{m}$. We also make use of the fact that the volume measure on $M$ is comparable to the Hausdorff $(N+m)$-measure, and the volume measure on level sets $\Phi^{-1}(y)$ is comparable to the Hausdorff $(N+m-n)$-measure on spherical shells $\left\{x: L^{-1} \leq|x| \leq L\right\}$ in $\mathbb{R}^{m}$ (with comparison constant depending on $L$ ).

The following proposition is an analytic version of Proposition 3.1, and a strengthening of Proposition 3.4 .

Proposition 3.8. There exists a constant $C>0$ so that for each $L>1$ we have

$$
\frac{1}{C L^{n}} \leq(J \Phi)_{\left(\pi_{0}, x_{0}\right)} \leq C L^{n}
$$

whenever $\pi_{0} \in O(n, m)$ and $x_{0} \in \mathbb{R}^{m}$ such that $L^{-1} \leq\left|x_{0}\right| \leq L$.

Proof. We derive the desired conclusion as a consequence of the Ahlfors regularity of the level sets. For two positive quantities $A$ and $B$, we write $A \approx B$ to mean that the ratio of $A$ and $B$ is bounded above and below by absolute constants depending only on the dimensions $m$ and $n$.

Let $p_{0}=\left(\pi_{0}, x_{0}\right) \in O(n, m) \times \mathbb{R}^{m}$ and assume that $L^{-1} \leq\left|x_{0}\right| \leq L$. Let $Q$ be a small cube centered at $p_{0}$ and contained in $O(n, m) \times\left(\mathbb{R}^{m}\right)^{*}$. By the continuity of the Jacobian $p \mapsto(J \Phi)_{p}$, by the coarea formula (3.1), and by Proposition 3.6, we have

$$
\begin{aligned}
(J \Phi)_{p_{0}} r^{N+m} & \approx(J \Phi)_{\left(\pi_{0}, x_{0}\right)} \mathcal{H}^{N+m}(Q) \approx \int_{Q}(J \Phi)_{p} d \mathcal{H}^{N+m}(p) \\
& \approx \int_{Q}(J \Phi)_{p} d p=\int_{\mathbb{R}^{n}}\left(\int_{\Phi^{-1}(y)} \chi_{Q}(q) d q\right) d y \\
& \approx \int_{\Phi(Q)} \mathcal{H}^{N+m-n}\left(\Phi^{-1}(y) \cap Q\right) d y \approx r^{N+m-n} \mathcal{H}^{n}(\Phi(Q)) .
\end{aligned}
$$

By Proposition 3.1, $\mathcal{H}^{n}(\Phi(Q)) \lesssim L^{n} r^{n}$ and $\mathcal{H}^{n}(\Phi(Q)) \gtrsim L^{-n} r^{n}$. The proof is complete.

\section{Proof of the Main theOREMS}

In this section we prove our main theorems, Theorem 1.4 and Theorem 1.3. We begin by recalling the notion of Sobolev mappings valued in metric spaces and the Morrey-Sobolev embedding theorem for supercritical Sobolev maps defined on John domains. Next, we prove a Morrey-Sobolev type estimate for maps from the product manifold $M=O(n, m) \times \mathbb{R}^{m}$ which factor through 
the evaluation map. The novelty here is that our estimate holds even for $W^{1, p}$ mappings with $p<N+m=\operatorname{dim} M$. Finally, we use such estimates to implement the scheme from [3] and establish Theorem 1.4. With a little more work, Theorem 1.3 follows as a corollary of Theorem 1.4.

Let $Y$ be a complete, separable metric space and let $\Omega$ be a domain in $\mathbb{R}^{n}$. A map $f: \Omega \rightarrow Y$ has a Borel function $g: \Omega \rightarrow[0, \infty]$ as an upper gradient if the inequality

$$
d_{Y}(f(\gamma(a)), f(\gamma(b))) \leq \int_{\gamma} g d s
$$

holds for every rectifiable curve $\gamma:[a, b] \rightarrow \Omega$. For the purposes of this paper, the Sobolev space $W^{1, p}(\Omega: Y)$ consists of all maps $f: \Omega \rightarrow Y$ which are $p$-integrable (e.g., $x \mapsto d_{Y}\left(f(x), y_{0}\right)$ is in $L^{p}(\Omega)$ for some fixed basepoint $\left.y_{0} \in Y\right)$ and which carry a $p$-integrable upper gradient. In fact, we will only work with continuous maps $f$; this entails no loss of generality for us since we restrict our attention to the supercritical case $p>n$. In general the $p$-integrability condition on $f$ may depend on the choice of basepoint $y_{0}$ (at least when $\Omega$ is unbounded), however, the integrability of $f$ will play no role in our arguments.

The definition of the $Y$-valued Sobolev space $W^{1, p}$ easily extends to the case when the domain is a Riemannian manifold.

For the next result, see, for example, [13, Theorem 9.7].

Theorem 4.1 (Morrey-Sobolev estimate on John domains). Let $\Omega$ be a domain in $\mathbb{R}^{n}$, let $p>n$, and let $Y$ be a complete separable metric space. For $A>0$ there exists a constant $C=C(n, p, A)$ so that

$$
\operatorname{diam} f(U) \leq C(\operatorname{diam} U)^{1-n / p}\left(\int_{U} g^{p}\right)^{1 / p}
$$

whenever $f$ is a continuous map in $W^{1, p}(\Omega: Y)$ with upper gradient $g \in L^{p}(\Omega)$, and $U \subset \Omega$ is a John domain with John constant A.

We are now ready to begin the proof of Theorem 1.4. Let $\Omega \subset \mathbb{R}^{n}$ be a bounded domain, let $p>n$ and let $f \in W^{1, p}(\Omega: Y)$ where $Y$ is a complete separable metric space. We may assume without loss of generality that $0 \notin \Omega$. By decomposing $\left(\mathbb{R}^{n}\right)^{*}$ into a countable disjoint family of spherical shells and using the countable subadditivity of Hausdorff measure and a dilation argument, we can assume without loss of generality that

$$
\Omega \subset B(0,2) \backslash B\left(0, \frac{1}{2}\right) .
$$

Hence the prior results established in this section and the previous section are applicable with $L=2$.

$$
\begin{gathered}
\text { Let } \Omega^{\prime}=\Phi^{-1}(\Omega) \subset O(n, m) \times \mathbb{R}^{m} \text { and define maps } F: \Omega^{\prime} \rightarrow Y \text { and } G: \Omega^{\prime} \rightarrow \mathbb{R} \text { by } \\
\qquad F=f \circ \Phi \quad \text { and } \quad G=g \circ \Phi \text {. }
\end{gathered}
$$

Then $F \in W^{1, p}\left(\Omega^{\prime}: Y\right)$ and $C G$ is an upper gradient for $F$ for some absolute constant $C$ (by the chain rule for upper gradients and Proposition 3.8). In fact, we will not explicitly make use of the Sobolev membership of $F$, but rather the following Morrey-Sobolev type inequality for $F$ which follows directly from its counterpart for $f$. Here we emphasize again that while $f$ is supercritical $(p>n), F$ need not be so (in general, we may have $p<N+m)$. Recall that $N=\operatorname{dim} O(n, m)$.

Proposition 4.2. There exists $C=C(n, m, p, \Omega)>0$ so that for all sufficiently small cubes $Q^{\prime} \subset \Omega^{\prime}$ we have

$$
\operatorname{diam} F\left(Q^{\prime}\right) \leq C\left(\operatorname{diam} Q^{\prime}\right)^{1-\frac{N+m}{p}}\left(\int_{Q^{\prime}} G(\pi, x)^{p} d \pi d x\right)^{1 / p} .
$$


Proof. By Proposition 3.3, there exists a constant $A>0$ depending only on $m$ so that $\Phi\left(Q^{\prime}\right)$ is a John domain with John constant $A$. By Theorem 4.1, we know that

$$
\operatorname{diam} f\left(\Phi\left(Q^{\prime}\right)\right) \leq C\left(\operatorname{diam} \Phi\left(Q^{\prime}\right)\right)^{1-\frac{n}{p}}\left(\int_{\Phi\left(Q^{\prime}\right)} g(y)^{p} d y\right)^{1 / p}
$$

for some $C=C(n, p, A)=C(n, m, p)$. Since $F=f \circ \Phi$ and $\operatorname{diam}\left(\Phi\left(Q^{\prime}\right)\right) \leq C \operatorname{diam} Q^{\prime}$, we conclude that

$$
\operatorname{diam} F\left(Q^{\prime}\right) \leq C\left(\operatorname{diam} Q^{\prime}\right)^{1-\frac{n}{p}}\left(\int_{\Phi\left(Q^{\prime}\right)} g(y)^{p} d y\right)^{1 / p} .
$$

In order to bring the integral of $G^{p}$ into the game, we shall further estimate the right hand side of (4.1) by using the co-area formula (3.1) applied to the map $\Phi$ and the $L^{p}$ function $G=g \circ \Phi$. To this end, we also employ Proposition 3.8 to control the Jacobian of $\Phi$. We estimate

$$
\begin{aligned}
\int_{Q^{\prime}} G(\pi, x)^{p} d \pi d x & \approx \int_{Q^{\prime}} G(\pi, x)^{p}(J \Phi)_{(\pi, x)} d \mathcal{H}^{N}(\pi) d \mathcal{H}^{m}(x) \\
& =\int_{O(n, m) \times \mathbb{R}^{m}} g(\Phi(\pi, x))^{p}(J \Phi)_{(\pi, x)} \chi_{Q^{\prime}}(\pi, x) d \mathcal{H}^{N}(\pi) d \mathcal{H}^{m}(x) \\
& \approx \int_{\mathbb{R}^{n}} \int_{\Phi^{-1}(y)} g(\Phi(\pi, x))^{p} \chi_{Q^{\prime}}(\pi, x) d \mathcal{H}^{N+m-n}(\pi, x) d \mathcal{H}^{n}(y) \\
& =\int_{\Phi\left(Q^{\prime}\right)} g(y)^{p} \mathcal{H}^{N+m-n}\left(\Phi^{-1}(y) \cap Q^{\prime}\right) d \mathcal{H}^{n}(y)
\end{aligned}
$$

Applying the Ahlfors $(N+m-n)$-regularity of the level sets $\Phi^{-1}(y)$ we conclude that

$$
\int_{Q^{\prime}} G(\pi, x)^{p} d \pi d x \approx\left(\operatorname{diam} Q^{\prime}\right)^{N+m-n} \int_{\Phi\left(Q^{\prime}\right)} g(y)^{p} d y .
$$

Combining (4.1) and (4.2) we obtained the desired conclusion.

With Proposition 4.2 in hand, Theorem 1.4 can be deduced by working in charts on the Riemannian manifold $O(n, m) \times\left(\mathbb{R}^{m}\right)^{*}$, or by directly reproducing the argument from [3]. For the sake of completeness, we briefly recapitulate part of this argument.

Proof of Theorem 1.4. Let $\gamma=\gamma(p, \alpha)$ be given as in (1.6). Suppose that the Stiefel exceptional set

$$
F_{\alpha}=\left\{\pi \in O(n, m): \mathcal{H}_{Y}^{\alpha}(f(\operatorname{im}(\pi) \cap \Omega))>0\right\}
$$

has positive $\mathcal{H}^{\gamma+\operatorname{dim} O(m)}$ measure. We may find a compact subset $C$ in $F_{\alpha}$ whose measure is positive and finite. By Frostman's lemma [18, Theorem 8.8], there exists a positive and finite Borel measure $\mu$ supported on $C$ satisfying the growth condition

$$
\mu(B(\pi, r)) \leq r^{\gamma+\operatorname{dim} O(m)}
$$

for all $\pi \in C$ and all $r>0$. Proposition 4.3 below allows us to conclude that $\mathcal{H}_{Y}^{\alpha}\left(F\left(\left(\{\pi\} \times \mathbb{R}^{m}\right) \cap\right.\right.$ $\left.\left.\Phi^{-1}(\Omega)\right)\right)=0$ for $\mu$-almost every $a \in C$. Since $\Phi\left(\{\pi\} \times \mathbb{R}^{m}\right)=\operatorname{im}(\pi)$, this contradicts the definition of $C$, and completes the proof of Theorem 1.4.

Proposition 4.3. Let $\Omega^{\prime}$ be a domain in $O(n, m) \times \mathbb{R}^{m}$. Let $E \subset O(n, m)$ be a set of finite $\mathcal{H}^{\gamma+\operatorname{dim} O(m)}$ measure and assume that $\mu$ is a positive Borel measure supported on $E$ satisfying the growth condition $\mu(B(\pi, r)) \leq r^{\gamma+\operatorname{dim} O(m)}$ for all $\pi \in O(n, m)$ and all $r>0$. Let $F \in W^{1, p}\left(\Omega^{\prime}: Y\right)$. Then $\mathcal{H}_{Y}^{\alpha}\left(F\left(\left(\{\pi\} \times \mathbb{R}^{m}\right) \cap \Phi^{-1}(\Omega)\right)\right)=0$ for $\mu$-almost every $a \in E$. 
Proposition 4.3 is analogous to [3, Proposition 3.2] and its proof is similar. Only one issue deserves further discussion. In the proof of Proposition 3.2 in [3] the equivalence of Hausdorff measure and dyadic Hausdorff measure is used to eliminate overlap between covering sets in the parameter space. We must deal with this issue in our setting also. We used the $\ell^{\infty}$ metric on the $\mathbb{R}^{m}$ factor precisely for this reason. On the $O(n, m)$ factor we could work locally in charts and use dyadic Hausdorff measures. Alternatively, and more simply, we can use the Besicovitch covering theorem to treat the issue of overlapping sets. Note that compact subsets of Riemannian manifolds admit a Besicovitch covering theorem [14, Example 1.15(c)]. We can modify the proof of Proposition 3.2 in [3], relaxing the dyadic assumption by appropriate use of a Besicovitch covering theorem. We leave the details to the reader.

Finally, we show how to deduce Theorem 1.3 from Theorem 1.4.

Proof of Theorem 1.3. The Grassmannian $G(n, m)$ identifies with the quotient $O(n, m) / O(m)$, where $O(m)$ acts on $O(n, m)$ by precomposition. In alternate language, $O(n, m)$ has the structure of an $O(m)$ bundle over $G(n, m)$. By the definition of the Stiefel and Grassmannian exceptional sets, we see that $F_{\alpha}$ is an $O(m)$ bundle over $E_{\alpha}$. Working in charts, we easily conclude that

$$
\operatorname{dim} F_{\alpha}=\operatorname{dim} E_{\alpha}+\operatorname{dim} O(m) .
$$

Furthermore, $E_{\alpha}$ is a null set for the $\mathcal{H}^{\gamma}$ measure on $G(n, m)$ (for some $\gamma>0$ ) if and only if $F_{\alpha}$ is a null set for the $\mathcal{H}^{\gamma+\operatorname{dim} O(m)}$ measure on $O(n, m)$. Theorem 1.4 allows us to conclude that $F_{\alpha}$ is a null set for $\mathcal{H}^{\gamma(p, \alpha)+\operatorname{dim} O(m)}$. Hence $E_{\alpha}$ is a null set for $\mathcal{H}^{\gamma(p, \alpha)}$. The proof is complete.

\section{A CONCLUding EXAmple}

In this final section we address the issue of sharpness in Theorem 1.3. Although we do not presently know whether or not the value of $\gamma(p, \alpha)$ in (1.6) is best possible, we can show that it is asymptotically sharp for $p$ near $n$. This can be seen in the following example.

Example 5.1. Fix integers $0<m<n$ and a line $L$ in $G(n, 1)$. The subset

$$
A(L):=\{V \in G(n, m): L \subset V\}
$$

can be identified with $G(n-1, m-1)$ as follows. Fix an element $f \in O(n, n-1)$ such that $\operatorname{im}(f)=L^{\perp}$, and define $\Theta: G(n-1, m-1) \rightarrow A(L)$ by $\Theta(W)=f(W) \oplus L$.

The map $\Theta$ is an isometry, when $A(L)$ is equipped with the subspace metric and the two Grassmannians are both equipped with any one of the metrics described in section 2 . This is easily seen by recalling that each of those metrics admits a representation in terms of principal angles. Note that all but one of the principal angles between $\Theta\left(W_{1}\right)$ and $\Theta\left(W_{2}\right)$ coincide with the principal angles between $W_{1}$ and $W_{2}$, while the remaining principal angle between $\Theta\left(W_{1}\right)$ and $\Theta\left(W_{2}\right)$ (corresponding to the direction $L$ which is common to both) is equal to zero.

In particular, the Hausdorff dimension of $A(L)$ is equal to $\operatorname{dim} G(n-1, m-1)=(m-1)(n-m)$.

Now fix a value $\epsilon, 0<\epsilon<\frac{n-m}{2 m-1}$, and set $p=n+\epsilon$. The universal upper bound for dimensions of images of $L$ under $W^{1, p}$ maps is

Set

$$
\frac{p \cdot 1}{p-n+1}=\frac{n+\epsilon}{1+\epsilon}
$$

$$
\alpha:=\frac{n+\epsilon}{1+2 \epsilon} .
$$

By [17, Theorem 3], there exists a map $f \in W^{1, p}\left(\mathbb{R}^{n}: \mathbb{R}^{n}\right)$ so that $\operatorname{dim} f(L) \geq \frac{n+\epsilon}{1+\epsilon}$, hence $\mathcal{H}^{\alpha}(f(L))>0$. By the choice of $\epsilon$, we have $\alpha>m$. In view of its definition, we see that $A(L)$ is a subset of the exceptional set $E_{\alpha}=\left\{V: \mathcal{H}^{\alpha}(f(V))>0\right\}$. Hence

$$
\operatorname{dim} E_{\alpha} \geq(m-1)(n-m) \text {. }
$$


In order to see how sharp is this example, we compare this value with the value of $\gamma(p, \alpha)$ coming from Theorem 1.3. For the data $p=n+\epsilon$ and $\alpha=\frac{n+\epsilon}{1+2 \epsilon}$ we have

$$
\operatorname{dim} E_{\alpha} \leq \gamma(p, \alpha)=m(n-m)-p\left(1-\frac{m}{\alpha}\right)=(m-1)(n-m)+(2 m-1) \epsilon .
$$

We conclude that the estimate in Theorem 1.3 is asymptotically sharp as $p \rightarrow n$.

It remains an open question to improve the quality of this example to obtain sharper bounds on the dimension and/or to increase the range of Sobolev exponents covered.

\section{REFERENCES}

[1] L. Ambrosio, N. Fusco, and D. Pallara. Functions of bounded variation and free discontinuity problems. Oxford Mathematical Monographs. Oxford University Press, New York, 2000.

[2] K. Astala. Area distortion of quasiconformal mappings. Acta Math., 173(1):37-60, 1994.

[3] Z. M. Balogh, R. Monti, and J. T. Tyson. Frequency of Sobolev and quasiconformal dimension distortion. J. Math. Pures Appl. (9), 99(2):125-149, 2013.

[4] Z. M. Balogh, J. T. Tyson, and K. Wildrick. Dimension distortion by Sobolev mappings in foliated metric spaces. Analysis and Geometry in Metric Spaces. To appear.

[5] Z. M. Balogh, J. T. Tyson, and K. Wildrick. Frequency of Sobolev dimension distortion of horizontal subgroups of Heisenberg groups. Preprint, 2013.

[6] A. Barg and D. Yu. Nogin. Bounds on packings of spheres in the Grassmann manifold. IEEE Trans. Inform. Theory, 48(9):2450-2454, 2002.

[7] C. J. Bishop and H. Hakobyan. Frequency of dimension distortion under quasisymmetric mappings. Preprint, 2012.

[8] J. H. Conway, R. H. Hardin, and N. J. A. Sloane. Packing lines, planes, etc.: packings in Grassmannian spaces. Experiment. Math., 5(2):139-159, 1996.

[9] F. W. Gehring. Rings and quasiconformal mappings in space. Trans. Amer. Math. Soc., 103:353-393, 1962.

[10] F. W. Gehring. The $L^{p}$-integrability of the partial derivatives of a quasiconformal mapping. Acta Math., 130:265$277,1973$.

[11] F. W. Gehring and J. Väisälä. Hausdorff dimension and quasiconformal mappings. J. London Math. Soc. (2), 6:504-512, 1973.

[12] J. C. Gower and G. B. Dijksterhuis. Procrustes problems, volume 30 of Oxford Statistical Science Series. Oxford University Press, Oxford, 2004.

[13] P. Hajłasz and P. Koskela. Sobolev met Poincaré. Mem. Amer. Math. Soc., 145(688):x+101, 2000.

[14] J. Heinonen. Lectures on analysis on metric spaces. Universitext. Springer-Verlag, New York, 2001.

[15] S. Hencl and P. Honzík. Dimension of images of subspaces under Sobolev mappings. Ann. Inst. H. Poincaré Anal. Non Linéaire, 29(3):401-411, 2012.

[16] O. Henkel. Sphere-packing bounds in the Grassmann and Stiefel manifolds. IEEE Trans. Inform. Theory, 51(10):3445-3456, 2005.

[17] R. P. Kaufman. Sobolev spaces, dimension, and random series. Proc. Amer. Math. Soc., 128(2):427-431, 2000.

[18] P. Mattila. Geometry of sets and measures in Euclidean spaces: fractals and rectifiability, volume 44 of Cambridge Studies in Advanced Mathematics. Cambridge University Press, Cambridge, 1995.

[19] Y.-C. Wong. Differential geometry of Grassmann manifolds. Proc. Nat. Acad. Sci. U.S.A., 57:589-594, 1967.

Mathematisches Institut, Universität Bern, Sidlerstrasse 5, 3012 Bern, Switzerland.

E-mail address: zoltan.balogh@math.unibe.ch

Department of Mathematics, Helsinki University, P.O. Box 68 (Gustaf Hällströminkatu 2B), Fi00014 Helsinki, Finland

E-mail address: pertti.mattila@helsinki.fi

Department of Mathematics, University of Illinois, 1409 West Green St., Urbana, IL 61801, USA.

E-mail address: tyson@math.uiuc.edu 